\title{
On boundedness of impulsively perturbed systems
}

\section{Sree Hari Rao}

\begin{abstract}
A few results on uniform and uniform ultimate boundedness properties of solutions of impulsively perturbed systems are presented in this paper, assuming the corresponding properties of solutions of the unperturbed nonlinear system.
\end{abstract}

\section{Introduction}

Liapunov's second method is a powerful tool for the qualitative analysis of differential systems. It has been extensively employed to study the stability and boundedness properties of solutions of ordinary differential systems (see [1], [4], [5]). But this method is not directly applicable for differential systems containing impulses. This is due to the fact that the solutions of the impulsively perturbed systems are discontinuous in nature, and this makes the study more interesting.

The main object of this paper is to extend Liapunov's second method to yield sufficient conditions for uniform and uniform ultimate boundedness of solutions of these systems.

\section{Preliminary results}

We use the same notations as given in [2].

We shall consider the measure differential equation

$$
D x=F(t, x)+p(t) D u,
$$

where $u: J \rightarrow R$ is a right continuous function of bounded variation on

Received 4 January 1978. 
every compact subinterval of $J$, and $p: J \rightarrow R^{n}$ is integrable with respect to $u$, and the discontinuities $t_{0}<t_{1}<t_{2}<\ldots<t_{k}<\ldots$ of $u$ tend to $\infty$ as $k$ tends to $\infty$. In (2.1), $D x$ and $D u$ denote the distributional derivatives of the functions $x$ and $u$ respectively, and Du can be identified with Stieltjes measure and will have the effect of suddenly changing the state of the system at the discontinuities of $u$. The equation (2.1) may be regarded as a perturbed system of the ordinary differential equation

$$
x^{\prime}=F(t, x),
$$

and thus is known as the impulsively perturbed system. For the stability properties of solutions of the equation (2.1), we refer the readers to [2] and [3].

Let $V(t, x)$ be a continuous function on $J \times R^{n}$ with values in $J$ and let the partial derivatives of $V$ be continuous. Then we define the derivative of $V$ with respect to the system (2.2) by

$$
\begin{aligned}
& V^{\prime}(t, x) \\
& (2.2)
\end{aligned}=\frac{\partial V}{\partial t}+\nabla V(t, x) \cdot F(t, x)
$$

where

$$
\nabla V=\left(\frac{\partial V}{\partial x_{1}}, \ldots, \frac{\partial V}{\partial x_{n}}\right)
$$

We adopt the following definitions from [4].

DEFINITION 2.1. A solution $x\left(t, t_{0}, x_{0}\right)$ of (2.2) is uniformly bounded if for any $\alpha>0$, there exists a $\beta(\alpha)>0$ such that the inequality $\left|x_{0}\right| \leq \alpha$ implies that $\left|x\left(t, t_{0}, x_{0}\right)\right|<\beta$ for all $t \geq t_{0}$.

DEFINITION 2.2. A solution $x\left(t, t_{0}, x_{0}\right)$ of (2.2) is uniformly ultimately bounded for bound $D$, if there exists $a, D>0$ and corresponding to any $\alpha>0$ there exists a $T=T(\alpha)>0$ such that the inequality $\left|x_{0}\right| \leq \alpha$ implies that $\left|x\left(t, t_{0}, x_{0}\right)\right|<D$ for all $t \geq t_{0}+T$.

\section{Main results}

Now we present our main results. 
THEOREM 3.1. Let $F(t, x) \in \bar{C}_{0}(x)$ and the solutions of (2.2) be uniformly bounded. Then the solutions of (2.1) are also uniformly bounded, provided

$$
\int_{t_{0}}^{\infty}|p(s)| d v_{u}(s)<\infty
$$

where $v_{u}$ is the total variation function of $u$.

Proof. Since the solutions of (2.2) are uniformly bounded and $F(t, x) \in \bar{C}_{0}(x)$, by Theorem 20.1 ([4], p. 105) there exists a Liapunov function $V(t, x)$ defined on $0 \leq t<\infty,|x| \geq R$, where $R>0$ may be large, satisfying

$$
a(|x|) \leq V(t, x) \leq b(|x|),
$$

where $a(p)$ and $b(p)$ are continuous increasing functions and $a(p) \rightarrow \infty$ as $r \rightarrow \infty$,

$$
V(t, x) \in \bar{C}_{0}(x)
$$

that is, $|\nabla V(t, x)| \leq L$, where $L$ is a positive constant, and

$$
V^{\prime}(t, x) \leq 0 \text {. }
$$

Now let $x(t)=x\left(t, t_{0}, x_{0}\right)$ be a solution of (2.1).

Following the proof of Theorem 3.1 of [2] and using (3.1), (3.2), (3.3), and Lemmas 2.4 and 2.5 of [2], we have for $t \geq t_{0}$,

$$
v(t, x(t)) \leq v\left(t_{0}, x_{0}\right)+L \int_{t_{0}}^{t}|p(s)| d v_{u}(s)
$$

Let $\alpha>0$ be given. Choose $\beta=\beta(\alpha)>0$ such that

$$
b(\alpha)+L M<a(\beta),
$$

where

$$
\int_{t_{0}}^{\infty}|p(s)| d v_{u}(s) \leq M<\infty
$$


Let $\left|x_{0}\right| \leq \alpha$. Then we claim that all the solutions of (2.1) are uniformly bounded. That is $\left|x\left(t, t_{0}, x_{0}\right)\right|<\beta$ for all $t \geq t_{0}$.

Suppose not. Then there exists a $t^{\prime} \in J$ such that

$$
\left|x\left(t^{\prime}\right)\right| \geq \beta
$$

whenever $\left|x_{0}\right| \leq \alpha$. From (3.4) for $t=t^{\prime}$ we have

$$
\begin{aligned}
a(\beta) \leq a\left(\left|x\left(t^{\prime}\right)\right|\right) \leq v\left(t^{\prime}, x\left(t^{\prime}\right)\right) & \leq v\left(t_{0}, x_{0}\right)+L \int_{t_{0}}^{t^{\prime}}|p(s)| d v_{u}(s) \\
& \leq b\left(\left|x_{0}\right|\right)+L \int_{t_{0}}^{\infty}|p(s)| d v_{u}(s) \\
& \leq b(\alpha)+L M<a(\beta),
\end{aligned}
$$

a contradiction, and this completes the proof.

The next theorem gives sufficient conditions for uniform ultimate boundedness of solutions of (2.1) for a bound $D$.

THEOREM 3.2. Let $F(t, x) \in \bar{C}_{0}(x)$ and the solutions of (2.2) be uniformly ultimately bounded for a bound $R^{\prime}$. Then the solutions of (2.1) are uniformly ultimately bounded for some bound $D$, provided

$$
\int_{t_{0}}^{\infty}|p(s)| d v_{u}(s)<\infty .
$$

Proof. Since the solutions of (2.2) are uniformly ultimately bounded for a bound $R^{\prime}$, in view of Theorem 20.4 ([4], p. 107) there exists a Liapunov function $V(t, x)$ defined on $0 \leq t<\infty$ and $|x| \geq R$, $0<R^{\prime}<R$, with the following properties:

$$
a(|x|) \leq V(t, x) \leq b(|x|),
$$

where $a(r)$ and $b(r)$ are continuous increasing functions, $a(r)>0$ for $r \geq R^{\prime}$ and $a(r) \rightarrow \infty$ as $r \rightarrow \infty$,

$$
V^{\prime}(t, x) \leq-C V(t, x)
$$

where $C$ is a positive constant, and

$$
V \in \bar{C}_{0}(x)
$$


From the relations (3.5), (3.6), and (3.7) it is clear that $|\nabla V(t, x)| \leq L$ and

$$
V^{\prime}(t, x) \leq-C a(R)
$$

Let $x(t)=x\left(t, t_{0}, x_{0}\right)$ be any solution of (2.1) existing to the right of $t_{0} \geq 0$.

Proceeding as in the above theorem we obtain for $t \geq t_{0}$,

$$
V(t, x(t)) \leq V\left(t_{0}, x_{0}\right)-C a(R)\left(t-t_{0}\right)+L \int_{t_{0}}^{t}|p(s)| d v_{u}(s)
$$

Let $\alpha>0$ be given. Select $T(\alpha)=\frac{b(\alpha)+L M}{C a(R)}$, where

$$
\int_{t_{0}}^{\infty}|p(s)| d v_{u}(s) \leq M<\infty
$$

Clearly $T$ depends only on $\alpha$.

We claim that all the solutions of (2.1) are uniformly ultimately bounded for a bound $D$. That is, for $D>0$ and corresponding to any $\alpha>0$ there exists a $T=T(\alpha)>0$ such that $\left|x_{0}\right| \leq \alpha$ implies $|x(t)|<D$ for all $t \geq t_{0}+T$.

Suppose not. Then there exists a $t^{\prime} \geq t_{0}+T$, such that

$$
\left|x\left(t^{\prime}\right)\right| \geq D \text {, }
$$

whenever $\left|x_{0}\right| \leq \alpha$. Therefore from (3.8) for $t=t^{\prime}$, we have

$$
\begin{aligned}
a(D) \leq a\left(\left|x\left(t^{\prime}\right)\right|\right) & \leq V\left(t^{\prime}, x\left(t^{\prime}\right)\right) \\
& \leq v\left(t_{0}, x_{0}\right)-C a(R)\left(t^{\prime}-t_{0}\right)+L \int_{t_{0}}^{\infty}|p(s)| d v_{u}(s) \\
& \leq b\left(\left|x_{0}\right|\right)-C a(R) T+L M \\
& \leq b(\alpha)-C a(R) T+L M=0
\end{aligned}
$$

a contradiction. Hence our claim is true. Therefore all the solutions of (2.1) are uniformly ultimately bounded for a bound $D$. This completes the 
proof.

REMARK 3.3. Suppose the perturbations are not impulsive; that is the state of the system changes continuously with respect to time; if the solutions of (2.2) are uniformly and uniformly ultimately bounded, then they are totally bounded (see [4], p. 120).

\section{References}

[1] Joseph La Salle and Solomon Lefschetz, Stability by Liapunov's direct method with applications (Mathematics in Science and Engineering, 4. Academic Press, New York, London, 1961).

[2] M. Rama Mohana Rao and V. Sree Hari Rao, "Stability of impulsively perturbed systems", Bulz. Austral. Math. Soc. 16 (1977), 99-110.

[3] V. Sree Hari Rao, "Stability of motion under impulsive perturbations" ( $\mathrm{PhD}$ thesis, Indian Institute of Technology, Kanpur, 1976).

[4] Taro Yoshizawa, Stability theory by Liapunov's second method (Publications of the Mathematical Society of Japan, 9. The Mathematical Society of Japan, Tokyo, 1966).

[5] T. Yoshizawa, Stability theory and the existence of periodic solutions cond almost periodic solutions (Applied Mathematical Sciences, 14. Springer-Verlag, New York, Heidelberg, Berlin, 1975).

Department of Mathematics,

Osmania University,

Hyderabad, Andhra Pradesh,

India. 